\title{
A study of kinematic cues and anticipatory performance in tennis using computational manipulation and computer graphics
}

\author{
Hirofumi Ida • Kazunobu Fukuhara • Seiji Kusubori • \\ Motonobu Ishii
}

Published online: 13 April 2011

(C) Psychonomic Society, Inc. 2011

\begin{abstract}
Computer graphics of digital human models can be used to display human motions as visual stimuli. This study presents our technique for manipulating human motion with a forward kinematics calculation without violating anatomical constraints. A motion modulation of the upper extremity was conducted by proportionally modulating the anatomical joint angular velocity calculated by motion analysis. The effect of this manipulation was examined in a tennis situation-that is, the receiver's performance of predicting ball direction when viewing a digital model of the server's motion derived by modulating the angular velocities of the forearm or that of the elbow during the forward swing. The results showed that the faster the server's forearm pronated, the more the receiver's anticipation of the ball direction tended to the left side of the serve box. In contrast, the faster the server's elbow extended, the more the receiver's anticipation of the ball direction tended to the right. This suggests that tennis
\end{abstract}

\footnotetext{
H. Ida $(\bowtie) \cdot K$. Fukuhara $\cdot$ M. Ishii

Department of Human System Science,

Graduate School of Decision Science and Technology,

Tokyo Institute of Technology,

Meguro, Tokyo, Japan

e-mail: hiroida@me.com

S. Kusubori

Department of Environmental Sciences,

Prefectural University of Hiroshima,

Hiroshima, Japan

H. Ida

Kanagawa Institute of Technology,

Human Media Research Center,

Atsugi, Kanagawa, Japan
}

players are sensitive to the motion modulation of their opponent's racket-arm.

Keywords Motion analysis - Motion modulation · Forward kinematics $\cdot \mathrm{CG} \cdot$ Digital human model

Humans can recognize various types of human motion instantaneously and accurately, not only when viewing the actions of real-life humans, but also when viewing the movements of artificial human models. For instance, the point-light display (PLD) technique has been one of the bestknown approaches and has shown perceptual robustness for biological motions (Johansson, 1973; Neri, Morrone, \& Burr, 1998). Previous studies have revealed that human perception is highly sensitive even to the PLD, in that observers were able to distinguish the gender of a walker (Kozlowski \& Cutting, 1977; Troje, 2002; Troje, Sadr, Geyer, \& Nakayama, 2006), identify friends by their walking pattern (Cutting \& Kozlowski, 1977), and recognize the emotions expressed by actors (Dittrich, Troscianko, Lea, \& Morgan, 1996; Pollick, Paterson, Bruderlin, \& Sanford, 2001).

Recently, in association with the development of computer graphics (CG) technology, various digital human models have become available for providing a visual stimulus of human motion. For example, the stickfigure model is regarded as a simplified human model, as is the point-light model, whereas many CG character models constructed from polygons or NURBS (non-uniform rational B-splines) imitate real humans. Motion capture systems and CG animation software facilitate the use of a digital human model in studies of human motion perception. One of the advantages of this approach is that several visual stimuli-for example, the polygon and stickfigure 
models - were created on the basis of the same motion data (Dekeyser, Verfaillie, \& Vanrie, 2002; Vanrie \& Verfaillie, 2004). These models were artificial versions of a real-world human or of one captured on video. They contained extra (pictorial) information in addition to the essential (kinematic) information that a point-light model does. It is necessary to evaluate the perceptual effect of digital human models, particularly in comparison with real-world or video displays. The anticipatory performance and visual search strategy when a tennis serve was viewed in order to predict the ball direction have been compared between a CG display with a polygon model and a video display (Fukuhara, Ida, Kusubori, \& Ishii, 2009). It was revealed that the polygon model allowed the observers to perceive anticipatory cues from the serve motion, but the response accuracy was lower than that of the video.

Digital human models have been utilized to simulate sports situations and to examine the response of the user in a virtual environment (VE) - for example, a baseball batter hitting a virtual ball (Gray, 2002), playing against a virtual pitcher (Ranganathan \& Carlton, 2007), or a handball goalkeeper facing a virtual thrower (Bideau, Multon, Kulpa, Fradet, Arnaldi, \& Delamarche, 2004; Vignais, Bideau, Craig, Brault, Multon, \& Kulpa, 2009). The aim was to examine the perception-action coupling in the sportsimulating VE, and this approach promises to reveal uncovered features that cannot be assessed in real-world situations or video displays.

The use of a digital human model has had another experimental advantage - namely, the arbitrary manipulation of displayed human motion. Principal component analysis has been used to identify systematic similarities and/or dissimilarities in the motion patterns across tennis passing shots (Huys, Smeeton, Hodges, Beek, \& Williams, 2008). The authors displayed simulated tennis shots in which motion dynamic structures were manipulated and revealed that tennis players predicted the shot direction by using the information in multiple lowdimensional dynamic modes. This approach was extended in further studies (Huys, Canal-Bruland, Hagemann, Beek, Smeeton, \& Williams, 2009; Williams, Huys, CanalBruland, \& Hagemann, 2009). In related work, spatially exaggerated tennis serve motions were displayed with a polygon model, and the exaggeration led to accurate categorizations of the types of tennis serves - that is, flat, slice, and topspin (Pollick, Fidopiastis, \& Braden, 2001). In other work, the joint motion of a runner was modulated, and the modulated motion was displayed using a polygon model and a stickfigure model (Hodgins, O'Brien, \& Tumblin, 1998). The results revealed that the identification of the running motion was more accurate for the polygon model than for the stickfigure model. The findings suggest that the perceptual performance for human motion could be affected by the pictorial ("geometric," according to the study) information of the human model. Their manipulation technique kinematically modified the motion data obtained from a dynamic simulation on the basis of the laws of physics and targeted the "torso rotation" and "dynamic arm motion" of the original running motion pattern.

In this study, we used another manipulation technique: The upper extremity motion was computationally manipulated by using a forward kinematics (FK) calculation, widely used in CG modeling and robotics, that outputs the position and orientation of the end effector as a function of joint angles (Zatsiorsky, 1998). This manipulation technique followed the anatomical constraints of the joint - that is, the degrees of freedom and range of motion-and calculated the motion data of the upper extremity as a function of the joint angular velocity modulated proportionally to that of the original motion. The computationally simulated test motion derived from the kinematic modulation is useful for revealing the quantitative relationship between the motion pattern of the performer and the perceptual performance of the observer. The anatomically validated motion can provide insight into the observer's perceptual skill for real-world human motion. Furthermore, the manipulation technique also enabled us to create various visual stimuli of $\mathrm{CG}$ animations in which the motion was arbitrarily modulated but anatomically feasible for humans.

Server motion and receiver cognition in tennis were examined in this study. A real-world tennis serve was analyzed and kinematically modulated at the server's racketarm and then was displayed with CG animations as visual stimuli for assessing the receiver's anticipatory performance. The kinematic modulation was performed by modulating the angular velocity of the forearm and that of the elbow during the forward swing phase of the original serve motion. The purposes of this study were to provide an explanation of the manipulation technique with an FK calculation and to verify whether the kinematic modulation has any effect on the receiver's anticipatory performance. We hypothesized that the anticipatory performance of the tennis player would be sensitive to the degree of motion modulation.

\section{Computation of motion analysis and motion modulation}

In the manipulation of the upper extremity, the anatomical joint angular velocity along an individual rotational axisfor example, the extension/flexion at the elbow-was selected as the modulating parameter. First, motion analysis was conducted to obtain every anatomical joint angular velocity in the upper extremity, using the three-dimensional coordinate data of the original motion. Second, motion 
modulation was performed using an FK calculation in which the obtained anatomical joint angular velocities were proportionally modulated. The computational expressions of the motion analysis and motion modulation will be described in the following sections.

\section{Motion analysis}

For motion analysis, the anatomical joint angular velocities at the wrist, forearm, elbow, and shoulder were calculated. The upper extremity was defined as a link segment model: The body segment of the hand, forearm, upper arm, and upper trunk are connected with the joint of the wrist, forearm, elbow, and shoulder (see Fig. 1). Note that the forearm was regarded as the joint as well as the body segment, because it has the degree of freedom of pronation/ supination. First, the resultant segment angular velocity was calculated from the motion data; then the resultant joint angular velocity at each joint was derived and, finally, separated into each anatomical axis of the joint as the anatomical joint angular velocity (Winter, 2005). An orthogonal reference frame (ijk) was defined for each body segment of the hand, forearm, upper arm, and upper trunk. The resultant segment angular velocity $\left(\omega^{\text {segment }}\right)$ is de-

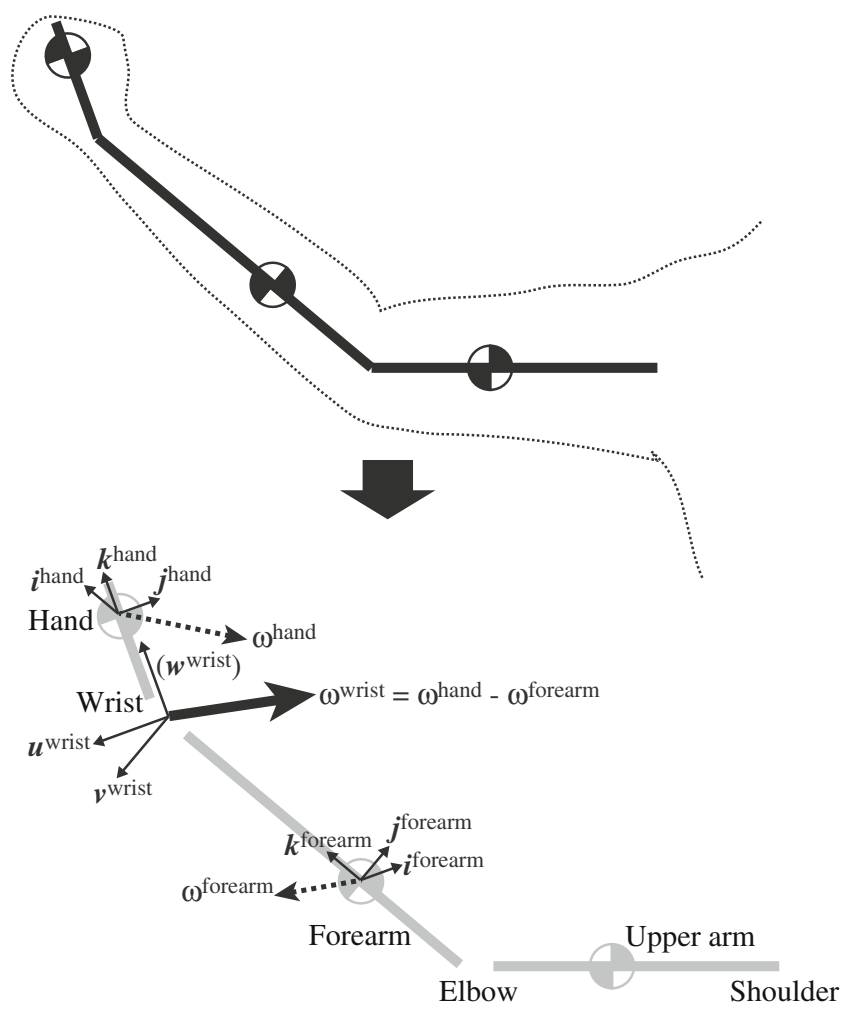

Fig. 1 Segment reference frame (e.g. $\boldsymbol{i}^{\text {hand }}, \boldsymbol{j}^{\text {hand }}, \boldsymbol{k}^{\text {hand }}$ ), joint reference frame (e.g. $\boldsymbol{u}^{\text {wrist }}, \boldsymbol{v}^{\text {wrist }}, \boldsymbol{w}^{\text {wrist }}$ ), resultant segment angular velocity (e.g. $\left.\omega^{\text {hand }}\right)$ and resultant joint angular velocity (e.g. $\left.\omega^{\text {wrist }}\right)$ scribed vectorially using the orthogonal vectors $i^{\text {segment }}$, $\boldsymbol{j}^{\text {segment }}$, and $\boldsymbol{k}^{\text {segment }}$, as follows:

$\omega^{\text {segment }}=\omega^{\prime}{ }_{1} i^{\text {segment }}+\omega^{\prime}{ }_{2} j^{\text {segment }}+\omega_{3}^{\prime} \boldsymbol{k}^{\text {segment }}$,

where $\omega_{1}^{\prime} \sim \omega_{3}^{\prime}$ are scalar. Since an arbitrary vector $\boldsymbol{r}$ that rotates around an axis with an angular velocity $\omega$ satisfies $d \boldsymbol{r} / d t=\omega \times \boldsymbol{r}$, transforming Eq. 1 for $\boldsymbol{r}=\boldsymbol{i}^{\text {segment }}, \boldsymbol{j}^{\text {segment }}$ or $\boldsymbol{k}^{\text {segment }}$, the values of $\omega_{1}^{\prime} \sim \omega_{3}^{\prime}$ are obtained as

$$
\begin{aligned}
\omega_{1}^{\prime} & =\boldsymbol{k}^{\text {segment }} \cdot \frac{d \boldsymbol{j}^{\text {segment }}}{d t}, \omega_{2}^{\prime}=\boldsymbol{i}^{\text {segment }} \cdot \frac{d \boldsymbol{k}^{\text {segment }}}{d t}, \omega_{3}^{\prime} \\
& =\boldsymbol{j}^{\text {segment }} \cdot \frac{d \boldsymbol{i}^{\text {segment }}}{d t},
\end{aligned}
$$

where the dot operator represents the scalar product. The resultant joint angular velocity $\left(\omega^{\text {joint }}\right)$ defined for the wrist, forearm, elbow, and shoulder is the relative angular velocity of the distal segment to the proximal segment of the joint:

$\omega^{\text {joint }}=\omega^{\text {distal segment }}-\omega^{\text {proximal segment }}$.

The joint reference frame was determined by an anatomical definition: palmar/dorsi flexion (plm/drs) and radial/ulnar flexion ( $\mathrm{rad} / \mathrm{uln})$ at the wrist; pronation/supination (prn/spn) at the forearm; flexion/extension (flx/ext) at the elbow; and horizontal adduction/abduction (had/hab), adduction/abduction (add/abd), and internal/external rotation (inr/exr) at the shoulder. The anatomical joint angular velocities $\left(\omega_{1}^{\prime} \sim \omega_{3}^{\prime}\right)$ were defined as the vector projection of the resultant joint angular velocity onto the orthogonal joint reference frame $(\boldsymbol{u v w})$ :

$$
\begin{gathered}
\omega_{1}=\omega^{\text {joint }} \cdot \boldsymbol{u}^{\text {joint }}, \quad \omega_{2}=\omega^{\text {joint }} \cdot \boldsymbol{v}^{\text {joint }}, \\
\omega_{3}=\omega^{\text {joint }} \cdot \boldsymbol{w}^{\text {joint }} .
\end{gathered}
$$

\section{Motion modulation}

For motion modulation, the motion of the upper extremity was manipulated by modulating the anatomical joint angular velocities and then calculating the resulting coordinate data of the joint,using an FK algorithm. The manipulation was defined such that it generated a displacement of the distal adjacent segment of the target joint but did not generate a displacement of the proximal adjacent segment. As a result, the modulation at a certain joint generated simultaneous displacements of all distal joints and segments; however, the positions and orientations of all proximal joints and segments remained unchanged. In particular, the position of the target joint was also unchanged.

The modulated anatomical joint angular velocity $\left(\omega_{\mathrm{m}}^{\text {mod_joint }}\right)$ was obtained by multiplying the original 
anatomical joint angular velocity for a certain duration (from $t_{0}$ to $t_{1}$ ) by a constant,

$\omega_{m}^{\text {mod_joint }}=\left\{\begin{array}{l}\omega_{m}\left(t<t_{0}\right) \\ C \omega_{m}\left(t_{0} \leq t<t_{1}\right), \\ \omega_{m}\left(t_{1} \leq t\right)\end{array}\right.$

$m=1,2,3$

where $C$ is an arbitrary constant associated with the modulation percentage and $m$ represents the nominal number of each anatomical axis. By using the anatomical description of the upper extremity, the modulated joint angular velocities ( $\left.\omega^{\text {mod_joint }}\right)$ are defined as follows:

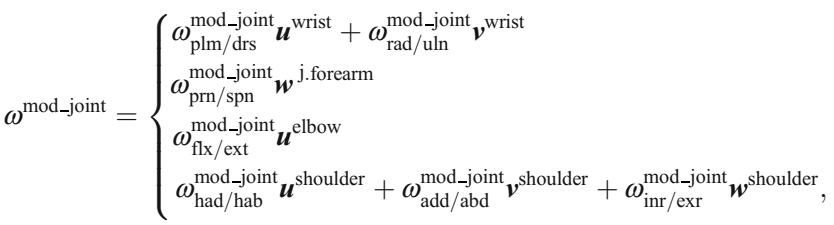

where $\mathrm{j}$. forearm represents the forearm joint in distinction from the forearm segment; joint motions with no degrees of freedom were omitted.

For general FK calculations, a coefficient matrix - that is, the Jacobian - is used to describe the relationship between the attitude of the end effector and the joint angular velocities of the link-segment system (Zatsiorsky, 1998):

$\frac{d \boldsymbol{r}}{d t}=\boldsymbol{J}(\alpha) \frac{d \alpha}{d t}$,

where $\boldsymbol{r}$ consists of the position vector and orientation vector of the end effector, $\boldsymbol{J}(\alpha)$ is the Jacobian, and $\alpha$ is the $n$th order group vector of the angular velocities of the $n$-joints. For the reconstruction of the modulated motion in this study, however, the coordinate data of all the upper extremity joints were required. Therefore, a sequential calculation of all the segment longitudinal unit vectors $\left(\boldsymbol{k}^{\text {segment }}\right)$ from the proximal joint to the distal joint was more convenient than using Eq. 7. The time derivative of $\boldsymbol{k}^{\text {segment }}$ is associated with $\omega^{\text {segment }}$ by the following equation:

$\frac{d \boldsymbol{k}^{\text {segment }}}{d t}=\omega^{\text {segment }} \times \boldsymbol{k}^{\text {segment }}$,

where the cross operator represents the vector product. The vector $\omega^{\text {segment }}$ was replaced by the modulated segment angular velocities ( $\left.\omega^{\text {mod_segment }}\right)$, which was calculated from $\omega^{\text {mod_joint }}$ in Eq. 6. This led to the following relationships:

\footnotetext{
$\omega^{\text {mod_upper arm }}=\omega^{\text {trunk }}+\omega^{\text {mod_shoulder }}$

$\omega^{\text {mod_forearm }}=\omega^{\text {mod_upper arm }}+\omega^{\text {mod_elbow }}$

$\omega^{\text {mod_hand }}=\omega^{\text {mod_forearm }}+\omega^{\text {mod_j.forearm }}+\omega^{\text {mod_wrist }}$.
}

We conducted a time progressive calculation to obtain the modulated segment longitudinal unit vectors $\left(\boldsymbol{k}^{\text {mod_segment }}\right)$ by using the differential transform of Eq. 8:

$$
\begin{aligned}
\boldsymbol{k}^{\text {mod_segment }}{ }_{(\tau)}= & 2 \Delta t\left(\omega^{\text {mod_segment }}{ }_{(\tau-1)}\right. \\
& \left.\times \boldsymbol{k}^{\text {mod_segment }}{ }_{(\tau-1)}\right) \\
& +\boldsymbol{k}^{\text {mod_segment }}{ }_{(\tau-2)},
\end{aligned}
$$

where $\Delta t$ is the time interval of the sampling frames and $\tau$, the frame number. Then the three-dimensional coordinate data of the modulated joint position ( $\left.x^{\text {mod joint }}\right)$ were obtained:

$\boldsymbol{x}^{\text {mod_elbow }}=\boldsymbol{x}^{\text {shoulder }}+\boldsymbol{k}^{\text {mod_upperarm }} l^{\text {upperarm }}$

$\boldsymbol{x}^{\text {mod_wrist }}=\boldsymbol{x}^{\text {mod_elbow }}+\boldsymbol{k}^{\text {mod_forearm }} l^{\text {forearm }}$

$\boldsymbol{x}^{\text {mod_MP }}=\boldsymbol{x}^{\text {mod_wrist }}+\boldsymbol{k}^{\text {mod_hand }} l^{\text {hand }}$,

where $l$ is a function of $\tau$ of the segment length determined by the original motion data and MP represents the third metacarpophalangeal joint.

The position and orientation of the racket were assumed to remain unchanged relative to those of the hand segment; therefore, the modulated racket reference frame $\left(\boldsymbol{R}^{\text {mod_racket }}\right)$ is described as

$\boldsymbol{R}^{\text {mod_racket }}=\boldsymbol{T} \boldsymbol{R}^{\text {mod_hand }}$,

where $\boldsymbol{T}$ is a transformation matrix dependent on $\tau$ that is not changed by the motion modulation and $\boldsymbol{R}^{\text {mod_hand }}$, the modulated hand reference frame. The coordinate data of the racket landmarks were determined by $\boldsymbol{R}^{\text {mod_racket }}$.

Non-modulated motion was derived through all the processes described above; namely, all the modulation constants in Eq. 5 were equal to one $(C=1)$. Note that the non-modulated motion also had a slight displacement, relative to the original captured motion, due to the reconstruction processing - for example, low-pass filtering and the assumption that the racket was rigid. At racket-ball impact, the increments of the kinematic parameters of the non-modulated motion, relative to the original motion, were $(\Delta X, \Delta Y, \Delta Z)=(0.003,0.011,0.006 \mathrm{~m})$ for the position of the racket face center, $\Delta V_{\mathrm{r}}=0.1 \mathrm{~m} / \mathrm{s}$ for the linear velocity of the racket face center, and $\Delta \alpha_{\text {yaw }}=0.5^{\circ}$ and $\Delta \alpha_{\text {pitch }}=-1.9^{\circ}$ for the racket attitude.

\section{Experiment with modulated motion}

Method

Participants Eight experienced male tennis players $(M \pm$ $S D$ : age $=21.1 \pm 2.1$ years, experience $=5.3 \pm 2.3$ years) participated in the experiment. The participants were 
college or graduate students who were carrying out daily practice in the collegiate club team, and all of them were unfamiliar with stimulus materials used for the experiment. They gave informed consent before the experiment.

Test stimuli A video-based system was used to collect three-dimensional coordinate data of serve motions on an outdoor tennis court. The serve motions performed by a skilled male test player (height $=1.73 \mathrm{~m}$, weight $=73 \mathrm{~kg}$, age $=23$ years, experience $=10$ years) were videotaped as the original motion at a $250-\mathrm{Hz}$ sampling rate, using two synchronized high-speed cameras (HSV-500C ${ }^{3}$, Nac Inc., Tokyo). The test player was instructed to hit a flat servethat is, a serve with less spin - using his best effort, aiming at a target area $(1 \times 1 \mathrm{~m})$ set at the back center of the court within the right serve box. Outrigger marker devices were attached to the test player's hand and wrist in order to calculate the anatomical angular velocities of the wrist flexion and forearm rotation separately (see Fig. 2). The most successful trial among the three collected flat serves was selected for analysis. The 21 landmarks of the entire body, 3 outrigger landmarks of the hand, 2 outrigger landmarks of the wrist, and 5 landmarks of the racket were manually digitized frame-by-frame, utilizing motion analysis software (Frame-DIAS II, DKH Inc., Tokyo). Then, using the process described in the Motion Analysis section, all the anatomical joint angular velocities of the racket-arm were obtained (see Fig. 3).

The modulation duration was set during the forward swing phase $(0.132 \mathrm{~s})$, beginning from the initiation of the forward swing $\left(t_{0}=\right.$ IFS) and ending at time of the racketball impact $\left(t_{1}=\right.$ Impact). The IFS was defined as the time at which the racket head was positioned at the lowest point. The forearm pronation/supination and elbow extension/ flexion were selected for modulation separately, because the joint angular velocities of them showed relatively high and consistently positive almost throughout the forward swing phase (Fig. 3b). The consistent positive sign of the joint angular velocity was interpreted as indicating typical unidirectional joint rotation of forearm pronation and elbow extension. The modulation coefficients were set to $C=0.7$, $0.85,1.15,1.3(-30 \%,-15 \%,+15 \%,+30 \%$, slow to fast modulation, respectively) for each joint rotation. Nonmodulated motion was also constructed through the same modulation procedure, with $C=1$ at every anatomical joint angular velocity. Finally, the test stimuli of serve animations consisted of one non-modulated motion $( \pm 0 \%)$, four forearm pronation/supination modulated motions $(-30 \%$, $15 \%,+15 \%,+30 \%$; see Fig. 4), and four elbow extension/ flexion modulated motions $(-30 \%,-15 \%,+15 \%,+30 \%$; see Fig. 5).

CG animations were created using CG modeling software (Maya 4.5, Alias Inc., Toronto). The template character "Jackie" from the Maya 4.5 Documentation and Lessons was used as the digital human model. The racket model was made of a rigid body polygon. A scripting language (Maya Embedded Language, MEL) was used to convert the motion data to the CG human model (Fukuhara, Ida, Kusubori, \& Ishii, 2009). The ball was erased from the animation to eliminate unexpected perceptual effects other than human motion; for example, the racket might miss the ball because the modulation procedure changed the path of the racket. The viewing point was set around the receiver's view point-that is, $1 \mathrm{~m}$ above the cross point of the baseline and sideline of the singles court on the right. The frame rate of the animation was set at $50 \mathrm{~Hz}$. The total duration of the animation was $1.6 \mathrm{~s}$ from the ready position to the racket-ball impact. The animation was occluded immediately after the frame of racket-ball impact.

Procedure The participants sat $3.5 \mathrm{~m}$ away from, and directly in front of, the screen on which the test stimuli were projected. The maximum height of the test serve motion was adjusted to $0.39 \mathrm{~m}$. This provided a visual angle of approximately $6.4^{\circ}$, which is similar to a real game situation. The experiment was conducted on our standalone test program built utilizing application development software (REAL Basic, ASCII Solutions Inc., Tokyo). This
Fig. 2 Attached hand marker and wrist marker (a), and digitizing points and calculated points (b) for original serve motion

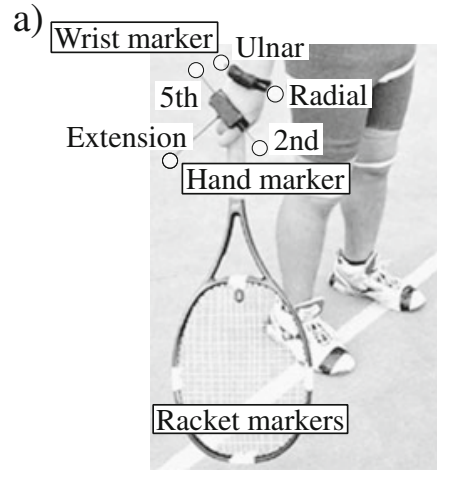

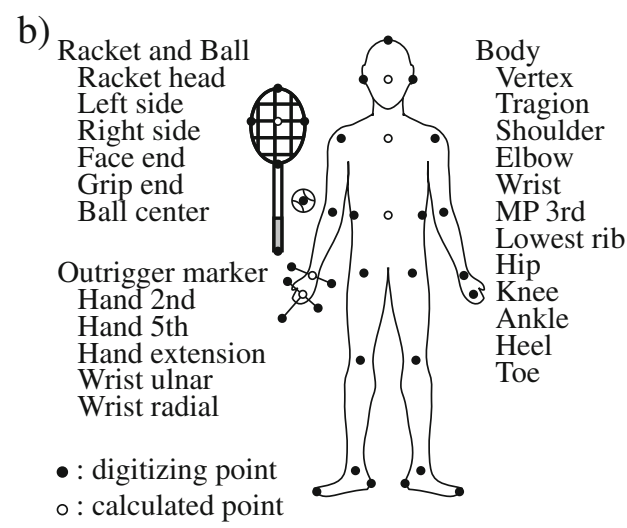



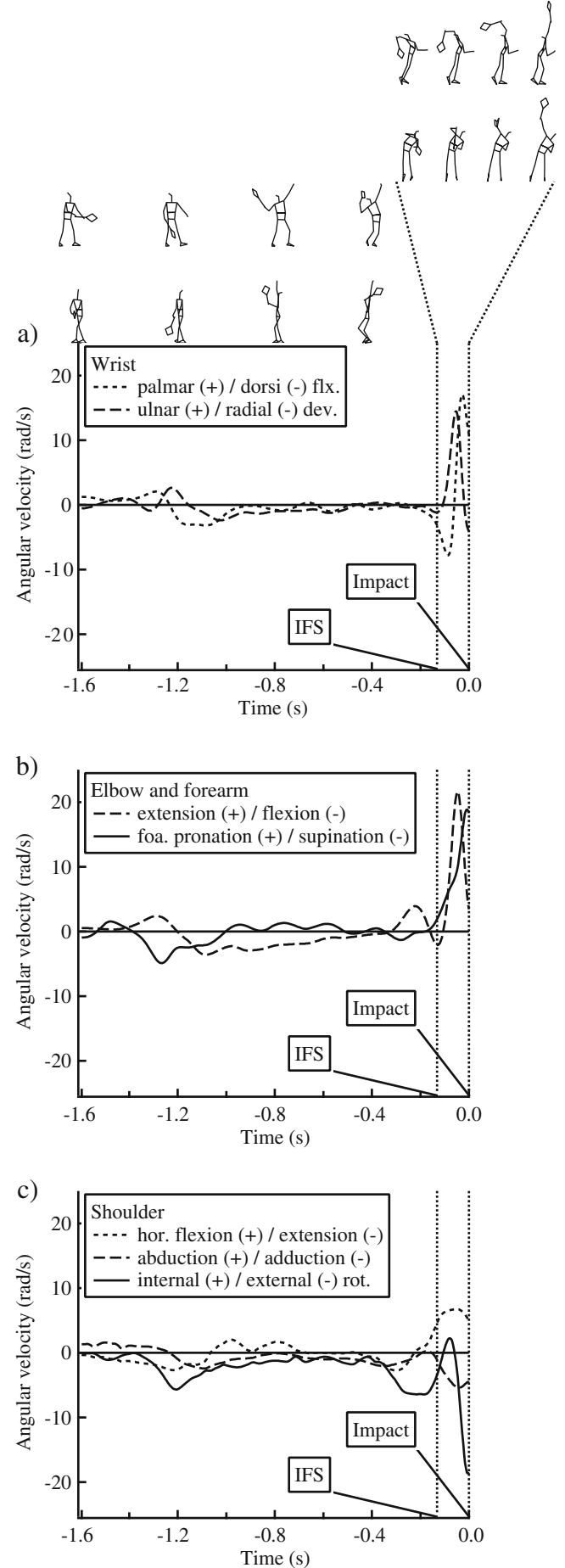

Fig. 3 The anatomical angular velocity of wrist (a), elbow and forearm (b) and shoulder (c). flx: flexion, dev: deviation, foa: forearm, hor: horizontal, and rot: rotation. IFS: Initiation of Forward Swing (-0.132 s), Impact: racket-ball impact

test program runs an experiment flow involving the presentation of a Quicktime movie, an input window for providing the anticipatory estimation score, and an auto-output function of all the response results. The participants first viewed the non-modulated motion at least three times and then viewed one of the modulated test motions, which included the non-modulated motion at a certain trial turn. After viewing, the participants were instructed to give their anticipatory score for the ball direction on a visual analogue scale (VAS) from 0 (left) to 100 (right) in comparison with the non-modulated motion ( 50 on VAS). The participants performed a total of 27 trials: 4 forearm modulated motion trials with 3 repeats, 4 elbow trials with 3 repeats, and 1 non-modulated trial with 3 repeats.

Data analysis The VAS scores were analyzed using statistical software (SPSS 17.0, SPSS Japan Inc., Tokyo). A repeated measure one-way analysis of variance (modulation percentage) with a least significant difference post hoc comparison was performed to test the effect of the modulation percentage on the anticipation of ball direction for the forearm modulation and elbow modulation. Mauchly's test of sphericity was conducted, and when there was a violation of the sphericity assumption, the Greenhouse-Geisser correction was used to adjust the degrees of freedom. Partial eta-squared $\left(\eta_{\mathrm{p}}{ }^{2}\right)$ was estimated to measure the effect size. The significance level was set at $\alpha=.05$.

\section{Result and discussion}

For forearm modulation, the anticipatory score of ball direction decreased (shifted to the left) monotonically with the modulation percentage (Fig. 6a). There was a significant effect on the modulation percentage, $F(4,28)=12.39$, $p<.01, \eta_{\mathrm{p}}{ }^{2}=.639$. Significant differences between modulation percentages were found in eight pairwise comparisons ( $p<.05$; *s in Fig. 6a). Because the forearm of the test server pronated throughout the forward swing phase (Fig. 3b), applying a positive modulation resulted in a faster pronating motion than non-modulated motion, whereas applying a negative modulation resulted in a slower pronating motion. The results indicate that the faster the server's forearm pronated, the more the anticipation of the ball direction tended to the left side of the serve box.

For elbow modulation, as opposed to forearm modulation, the anticipatory score of ball direction increased(shifted to the right) monotonically with the modulation percentage (Fig. 6b). There was a significant effect on the modulation percentage, $F(1.56,28)=7.05$, $p<.01, \eta_{\mathrm{p}}{ }^{2}=.502$. Significant differences between modulation percentages were found in five pairwise comparisons $\left(p<.05 ; *^{*}\right.$ in Fig. 6b). The server's elbow extended almost throughout the forward swing phase (Fig. 3b). Using the same consideration as forearm modulation, the result signified that the faster the server's 


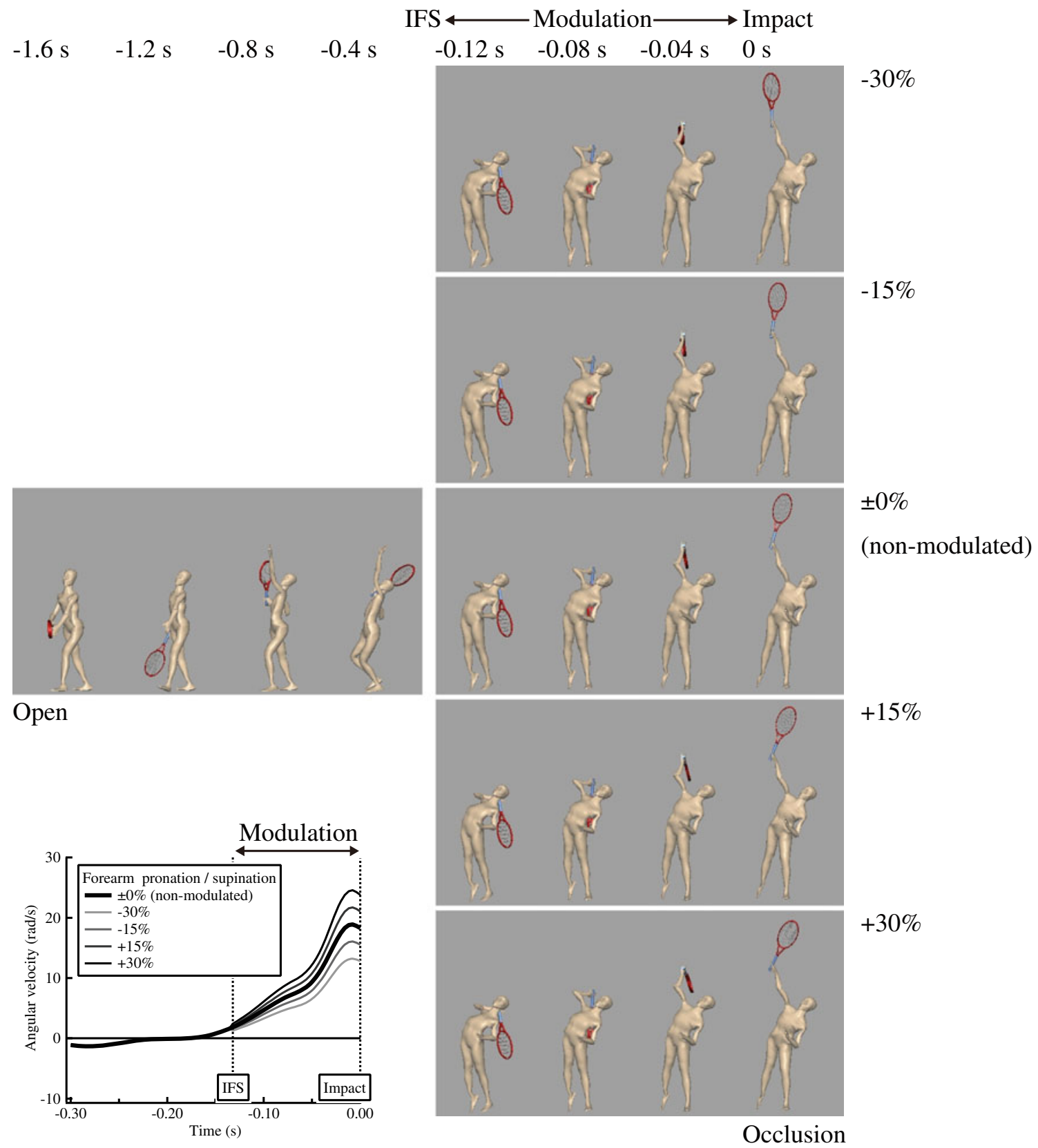

Fig. 4 The sequential image of CG animation with the modulation of the forearm angular velocity. Time-course curves show the profile of the modulated forearm pronation $(+)$ / supination (-) angular velocity

elbow extended, the more the anticipation of ball direction tended to the right.

These results suggest that tennis players are sensitive to the motion modulation of their opponent's racket-arm. In previous work, the adjustment of preimpact racket motion during overhand serve- for example, changing the racket's vertical and lateral velocity-was carried out by skilled players when going from the first to the second serve (Chow, Carlton, Lim, Chae, Shim, Kuenster, \& Kokubun, 2003); therefore, receivers might have changed the anticipatory judgment depending on the opponent's arm kine- matics. It is highly possible that skilled players may benefit from the visual information found in the racket-arm and racket during the forward swing and use it as an anticipatory cue.

The results demonstrated that the manipulation technique worked well for detecting the change in the pattern of the anticipation of ball direction. Interestingly, both forearm modulation and elbow modulation evoked monotonic tendencies in ball direction score depending on modulation percentage. Both of these target motions were almost unidirectional, forearm pronation or elbow 


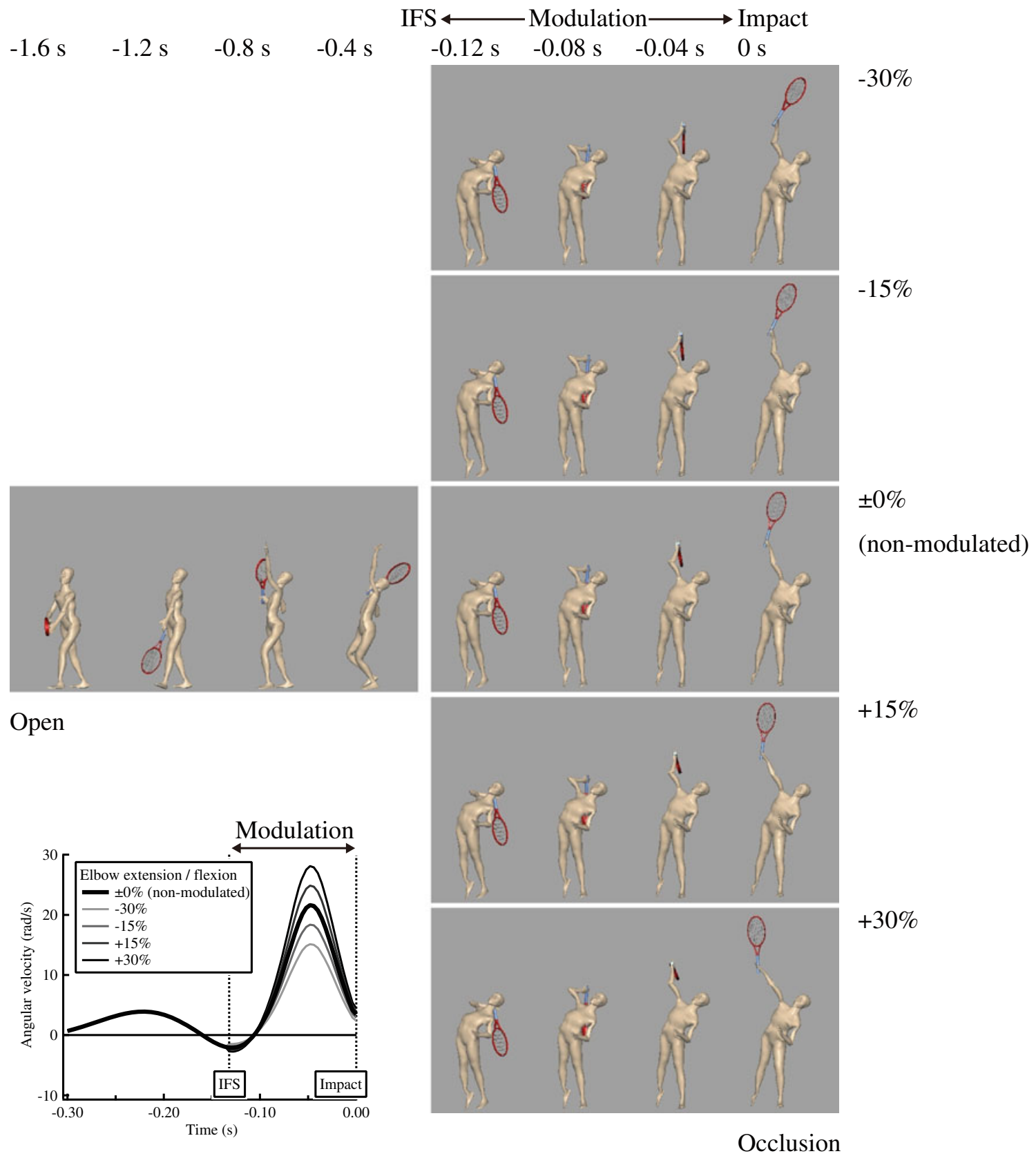

Fig. 5 The sequential image of CG animation with the modulation of the elbow angular velocity. Time-course curves show the profile of the modulated elbow extension (+) / flexion (-) angular velocity

extension, in the motion analysis; therefore, the result is clear: The anticipation of ball direction shifts monotonically depending on joint motion speed. The situation would be more complex if the modulation targeted bidirectional motion-for example, the dorsi flexion followed by the palmar flexion - or multijoint motions. The judgment of ball direction is one of the most popular criteria for assessing the response accuracy in sports situations, and thus, the manipulation technique contributes further to this field.

\section{General discussion}

We proposed a methodological approach that utilized the manipulation technique with the FK calculation to control test motion quasi-arbitrarily and a CG display with a digital human model. This methodology is referred to by Hodgins et al. (1998). However, our approach was intended to be more applicable to ball sport practitioners; therefore, we examined the anticipatory performance of a tennis opponent's serve motion, which requires great attention and 
Fig. 6 The anticipatory score $(M \pm S D)$ of estimated ball direction for forearm modulation (a) and elbow modulation (b) .

$*: p<.05$ a) Forearm modulation

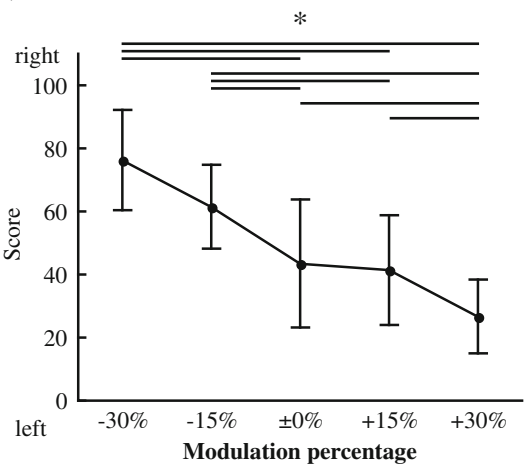

b) Elbow modulation

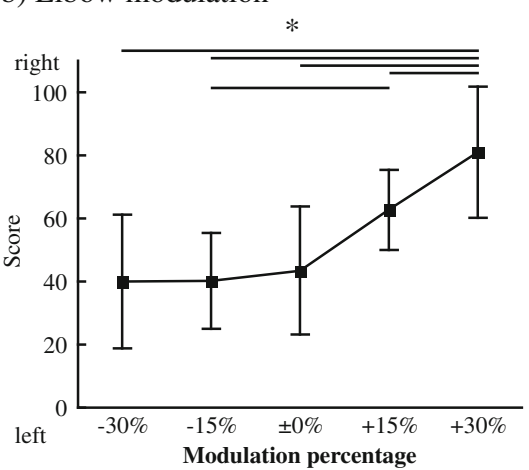

skilled visual search behavior. The motion exaggeration in the spatial location enhances the discrimination accuracy of the type of tennis serves (Pollick, Fidopiastis, \& Braden, 2001). This technique has modified the joint positions of the entire body but did not permit precise manipulation of the anatomical joint rotation. The use of anatomically validated motion in our study enables meaningful suggestions for practitioners, because real players may duplicate such motions without violating anatomical constraints. Our manipulation technique is more plausible in an anatomical sense, but this still presents a problem: Can real players hit the ball with the manipulated motion?

During the anticipation of the tennis ground stroke, the skilled players spent a significantly shorter time visually searching the racket area than did their less skilled counterparts, whereas no differences were observed between them at the racket-arm and hand area (Williams, Ward, Knowles, \& Smeeton, 2002). This suggests that the changes in racket kinematics may have a greater influence on less skilled players than on skilled ones. However, gaze analysis gave no conclusive evidence about whether or not the racket or racket-arm is more informative; to understand this, it will be necessary to clarify the effect of central vision and peripheral vision in tennis perception. In a related study, the isolated motion display of a badminton stroke revealed that the PLD of the racket-arm raised the anticipatory performance of experts above chance level at an earlier phase than that of the racket (Abernethy \& Zawi, 2007). The challenge is determining whether or not anticipatory performance based on the racket or racket-arm depends on task or skill level. In addition, the anticipatory accuracy for the PLD of the lower extremity cleared chance level at an even earlier phase than that of the racket-arm. Skilled players appear to benefit from the anticipatory cue located around the proximal body; in contrast, less skilled players preferred to focus on more deterministic cues located around the racket area (Williams et al., 2002).
The limitations of this study were as follows: The relationship with real world, film, or PLD was not studied, and the VAS response could not explain the effect of perception-action coupling. Recently, the effect of using a digital human model for studying the anticipatory performance of a tennis serve was discussed, and a CG display was found to be more similar to a video display rather than to a PLD (Fukuhara et al., 2009). The assessment of a CG display of the manipulated motion in comparison with real world or film remains to be examined.

In our study, a video-based system was used to capture the motion of the test player because the field was a wide outdoor environment. Auto-motion analysis systems-for example, MotionAnalysis (MotionAnalysis Corp., Santa Rosa, CA) or VICON (Vicon Inc., Los Angeles, CA) - are also available if the environment is suitable for data collection. These systems simplify the process of creating visual stimuli.

Previous studies (Abernethy, 1990; Ward, Williams, \& Bennett, 2002; Williams et al., 2002) have suggested that skilled players were able to perceive anticipatory cues from an earlier part of an opponent's stroke motion and more proximal segments of the opponent's body than were their unskilled counterparts. Our motion modulation, in contrast, focused on the later part (forward swing phase) and more distal segments (racket and racket-arm), because early and proximal modulation might generate complicated and unexpected motion. If the motion modulation with $\mathrm{FK}$ at the early phase and proximal segment generates the visual stimuli of natural and valid modulated motion, the study using this will provide mutually complementary understandings with those previous studies.

The methodology of this study included a distal approach in cognitive psychology, but it allowed information to be added in parallel to the visual stimulus. A typical distal approach uses natural motion as a visual stimulus and subtracts information (e.g., color, shape) from the motion. Using simplified information from the CG model for a captured natural motion is subtractive, and thus, this 
approach is categorized as a distal approach. However, the motion modulation is additive, which is a typical concept in the proximal approach, because the parameters of the modulated joint angular velocity are added to control the racket-arm motion. Pollick et al. (2001) referred to this distal but additive approach as unique in the study of biological motion perception. The motion manipulation has a significant effect on biological motion perception, and the motion modulation with FK calculation may have various applications in the study of human motion perception, together with the techniques such as CG animation or motion capture (e.g., in a sport simulation or in nonverbal communication).

\section{References}

Abernethy, B. (1990). Anticipation in squash: Differences in advance cue utilization between expert and novice players. Journal of Sports Sciences, 8, 17-34.

Abernethy, B., \& Zawi, K. (2007). Pickup of essential kinematics underpins expert perception of movement patterns. Journal of Motor Behavior, 39, 353-367.

Bideau, B., Multon, F., Kulpa, R., Fradet, L., Arnaldi, B., \& Delamarche, P. (2004). Using virtual reality to analyze links between handball thrower kinematics and goalkeeper's reactions. Neuroscience Letters, 372, 119-122.

Chow, J., Carlton, L. G., Lim, Y.-T., Chae, W.-S., Shim, J.-H., Kuenster, A., et al. (2003). Comparing the pre- and post-impact ball and racquet kinematics of elite tennis players' first and second serves: A preliminary study. Journal of Sports Sciences, 21, 529-537.

Cutting, J. E., \& Kozlowski, L. T. (1977). Recognizing friends by their walk: Gait perception without familiarity cues. Bulletin of the Psychonomic Society, 9, 353-356.

Dekeyser, M., Verfaillie, K., \& Vanrie, J. (2002). Creating stimuli for the study of biological-motion perception. Behavior Research Methods, Instruments, \& Computers, 34, 375-382.

Dittrich, W. H., Troscianko, T., Lea, S. E., \& Morgan, D. (1996). Perception of emotion from dynamic point-light displays represented in dance. Perception, 25, 727-738.

Fukuhara, K., Ida, H., Kusubori, S., \& Ishii, M. (2009). Anticipatory judgment of tennis serve: A comparison between video images and computer graphics animations. International Journal of Sport and Health Science, 7, 12-22.

Gray, R. (2002). Behavior of college baseball players in a virtual batting task. Journal of Experimental Psychology. Human Perception and Performance, 28, 1131-1148.
Hodgins, J. K., O’Brien, J. F., \& Tumblin, J. (1998). Perception of human motion with different geometric models. IEEE Transactions on Visualization and Computer Graphics, 4, 307-316.

Huys, R., Canal-Bruland, R., Hagemann, N., Beek, P. J., Smeeton, N. J., \& Williams, A. M. (2009). Global information pickup underpins anticipation of tennis shot direction. Journal of Motor Behavior, 41, $158-171$.

Huys, R., Smeeton, N. J., Hodges, N. J., Beek, P. J., \& Williams, A. M. (2008). On the dynamic information underlying visual anticipation skill. Perception \& Psychophysics, 70, 1217-1234.

Johansson, G. (1973). Visual perception of biological motion and a model for its analysis. Perception \& Psychophysics, 14, 201-211.

Kozlowski, L. T., \& Cutting, J. E. (1977). Recognizing the sex of a walker from a dynamic point-light display. Perception \&Psychophysics, 21, 575-580.

Neri, P., Morrone, M. C., \& Burr, D. C. (1998). Seeing biological motion. Nature, 395, 894-896.

Pollick, F. E., Fidopiastis, C., \& Braden, V. (2001). Recognising the style of spatially exaggerated tennis serves. Perception, 30, 323-338.

Pollick, F. E., Paterson, H. M., Bruderlin, A., \& Sanford, A. J. (2001). Perceiving affect from arm movement. Cognition, 82, B51-B61.

Ranganathan, R., \& Carlton, L. G. (2007). Perception-action coupling and anticipatory performance in baseball batting. Journal of Motor Behavior, 39, 369-380.

Troje, N. F. (2002). Decomposing biological motion: A framework for analysis and synthesis of human gait patterns. Journal of Vision, 2(5, Art. 2), 371-387.

Troje, N. F., Sadr, J., Geyer, H., \& Nakayama, K. (2006). Adaptation aftereffects in the perception of gender from biological motion. Journal of Vision, 6(8, Art. 7), 850-857.

Vanrie, J., \& Verfaillie, K. (2004). Perception of biological motion: a stimulus set of human point-light actions. Behavior Research Methods, Instruments, \& Computers, 36(4), 625-629.

Vignais, N., Bideau, B., Craig, C., Brault, S., Multon, F., \& Kulpa, R. (2009). Virtual environments for sport analysis: perception-action coupling in handball goalkeeping. International Journal of Virtual Reality, 8(4), 43-48.

Ward, P., Williams, A. M., \& Bennett, S. J. (2002). Visual search and biological motion perception in tennis. Research Quarterly for Exercise and Sport, 73, 107-112.

Williams, A. M., Huys, R., Canal-Bruland, R., \& Hagemann, N. (2009). The dynamical information underpinning anticipation skill. Human Movement Science, 28, 362-370.

Williams, A. M., Ward, P., Knowles, J. M., \& Smeeton, N. J. (2002). Anticipation skill in a real-world task: measurement, training, and transfer in tennis. Journal of Experimental Psychology. Applied, $8,259-270$.

Winter, D. A. (2005). Biomechanics and motor control of human movement (3rd ed.). Hoboken: Wiley.

Zatsiorsky, V. M. (1998). Differential kinematics of human movement Kinematics of human motion. Champaign: Human Kinetics. 